\title{
Effects of nonlocality in time of interactions of an atom with its surroundings on the broadening of spectral lines of atoms
}

\author{
Renat Kh. Gainutdinov ${ }^{\text {a }}$ Aigul A. Mutygullina ${ }^{a}$ \\ Werner Scheid ${ }^{b}$ \\ ${ }^{a}$ Kazan State University, Department of Physics, Kremlevskaya st.,18, Kazan, \\ Russia \\ ${ }^{\mathrm{b}}$ Institut für Theoretische Physik der Justus-Liebig-Universität, D-35392, Giessen, \\ Germany
}

\begin{abstract}
We investigate effects of nonlocality in time of the interaction of an atom with its surroundings on the spectral line broadening. We show that these effects can be very significant: In some cases nonlocality in time of this interaction can give rise to a spectral line splitting.
\end{abstract}

PACS: 03.65.Bz; 32.70.Jz; 31.70.Hq.

Key words: Nonlocal-in-time interaction, open quantum systems, spectral line broadening

Separation of scales plays an important role in many problems of physics because it allows one to select relevant degrees of freedom and to describe a quantum system only in their terms. Integrating out other degrees of freedom results in nonlocality in time of the interaction in this system. As is well known, the dynamics of such a system is not governed by the Schrödinger equation, since this equation is local in time, and interaction Hamiltonians describe instantaneous interactions. At the same time, in Ref. [1] it has been shown that the Schrödinger equation is not the most general dynamical equation consistent with the current concepts of quantum physics, and a more general equation of motion has been derived as a consequence of the Feynman $[2,3]$ and canonical approaches to quantum theory. Being equivalent to the Schrödinger equation in the case of instantaneous interactions, this generalized dynamical equation permits the generalization to the case where the dynamics of a quantum system is generated by a nonlocal-in-time interaction. 
It has been shown [1] that the generalized quantum dynamics (GQD) developed in this way may be an important tool for solving various problems in quantum theory.

An invaluable tool for computing physical quantities in the theories with disparate energy scales are effective field theories (EFT's) [4,5]. Following the pioneering work of Weinberg [6], the EFT of nuclear forces has become very popular in nuclear physics (for a rewiew, see [7]). To describe low energy processes involving nucleons and pions, all operators consistent with the symmetries of QCD are included in an effective Lagrangian. A fundamental difficulty is that such Lagrangians yield graphs which are divergent, and give rise to singular quantum mechanical potentials. These potentials do not satisfy the requirements of ordinary quantum mechanics and need to be regulated, and renormalization must be performed. In this way one can successfully perform calculations of many quantities in nuclear physics. However, in such a way one cannot parametrize the $N N$ interaction by using some Hamiltonian, and there are not any equations for renormalized amplitudes in subtractive EFT's. As a result, in nuclear physics the Schrödinger equation is not valid even in the nonrelativistic limit. Meanwhile, as has been shown in [1], only the generalized dynamical equation must be valid in all the cases, not the Schrödinger equation. In Ref. [8] it has been shown that, in fact, low energy nucleon dynamics in the EFT of nuclear forces is governed by the generalized dynamical equation with nonlocal-in-time interaction operator when this equation is not equivalent to the Schrödinger equation. Moreover, in leading order this dynamics is just the same as in the case of the model $[1,9]$ developed as a test model illustrating the possibility of the extension of quantum dynamics provided by the formalism of the GQD. This shows the predictive power of the GQD: In Refs. [10,11] it has been shown that the existence of the quark and gluon degrees of freedom should give rise to the fact that low energy nucleon dynamics is governed by a nonlocal-in-time interaction operator. In the case where the interaction is separable the form of this operator is uniquely determined by the corresponding form factor [1]. At the same time, as it follows from the general analysis of the diagrams of the EFT, the leading order two nucleon $T$ matrix is separable with the unite form factor. Thus the GQD predicts, without summing diagrams and performing procedures of regularization and renormalization, that the leading order two nucleon $T$ matrix in the EFT approach should be the same as in the above model with the unite form factor, and this really takes a place. This predictive power of the GQD is a result of the fact that the generalized dynamical equation, which forms the basis of this formalism, has been derived in Ref. [1] as the most general dynamical equation consistent with the current concepts of quantum physics.

The above gives us the expect that the GQD can be applied in the theory of open quantum system where the separation of scales also plays an important role. In this theory one consider an open system in contact with its surround- 
ings consist of a practically infinite number of degrees of freedom and act as a whole identify, referred as the thermal bath, on the open system. It is assumed that the time scale of the open system is very long compared with the relaxation time of the bath, but shorter than the recurrence time for the whole system considered a closed system. For describing the dynamics of open quantum systems one can start with a closed system. A reduction to a small subsystem (open system) produces nonlocal-in-time equations. The application of these equations to real situations is extremely complex. For this reason it is natural to describe the evolution of an open system only in terms of its degrees of freedom by using a dynamical equation derived from the first principles of quantum theory. Such a program has been realized, for example, in the Lindblad theory $[12,13]$. The possibility of the application of this theory to some practical problems was considered, for example, in the works [14-17]. However, the dynamical equation of this theory is a Markovian-type equation, i.e., it does not take into account nonlocality of the interaction in time. Note, in this connection, that the Markovian assumption is usually used to overcome the above mentioned difficulties in the theory. For this reason Lindblad theory is based on the axioms which imply that the theory is local in time. This allows one to derive, as a consequence of the general principles, an equation, which governs the evolution of an open system, without entering into details of the physics of the external system. The aim of the present paper is to show that, keeping these advantages of Lindblad's theory, the GQD allows one to describe the evolution of open quantum systems without using the Markovian assumption. By using the example of a two-level atom interacting with its environment, we will investigate the effects of the nonlocality in time of the interaction on the character of the dynamics of an open system.

Let us briefly consider the main features of the GQD. The basic concept of the canonical formalism of quantum theory is that it can be formulated in terms of vectors of a Hilbert space and operators acting on this space. The postulates establish the connection between the vectors and operators and states of a quantum system and observables, and prescribe how the probability of an event should be computed (see, for example [18]). In the canonical formalism these postulates are used together with the dynamical postulate according to which the time evolution of a quantum system is governed by the Schrödinger equation. In the equivalent Feynman formalism quantum theory is formulated in terms of probability amplitudes without resorting to vectors and operators acting on a Hilbert space. In this approach the following assumption is used as a basic postulate:

The probability of an event is the absolute square of a complex number called the probability amplitude. The joint probability amplitude of a time-ordered sequence of events is the product of separate probability amplitudes of each of these events. The probability amplitude of an event which can happen in several different ways is a sum of the probability amplitudes for each of these 
ways.

In particular, $\left\langle\psi_{2}\left|U\left(t, t_{0}\right)\right| \psi_{1}\right\rangle$, being the probability of finding, the quantum system in the state $\left|\psi_{2}\right\rangle$ in a measurement at time $t$ if at time $t_{0}$ it was in the state $\left|\psi_{1}\right\rangle$, can be represented as a sum of contributions from all alternative ways of realization of the corresponding evolution process. Dividing these alternatives in different classes, we can then analyze such a probability amplitude in different ways [3]. For example, subprocesses with definite instants of the beginning and end of the interaction in the system can be considered as such alternatives. In this way the amplitude $\left\langle\psi_{2}\left|U\left(t, t_{0}\right)\right| \psi_{1}\right\rangle$ can be written in the form [1]

$$
\left\langle\psi_{2}\left|U\left(t, t_{0}\right)\right| \psi_{1}\right\rangle=\left\langle\psi_{2} \mid \psi_{1}\right\rangle+\int_{t_{0}}^{t} d t_{2} \int_{t_{0}}^{t_{2}} d t_{1}\left\langle\psi_{2}\left|\tilde{S}\left(t_{2}, t_{1}\right)\right| \psi_{1}\right\rangle
$$

where $\left\langle\psi_{2}\left|\tilde{S}\left(t_{2}, t_{1}\right)\right| \psi_{1}\right\rangle d t_{1} d t_{2}$ is the probability amplitude that at time $t_{1}$ the system in the state $\left|\psi_{1}\right\rangle$, then the interaction in the system begins in the time interval $\left(t_{1}, t_{1}+d t_{1}\right)$ and ends in the time interval $\left(t_{2}, t_{2}+d t_{2}\right)$ and at time $t_{2}+d t_{2}$ the system will be in the state $\left|\psi_{2}\right\rangle$. Eq. (1) is a consequence of the first postulate of Feynman's approach to the quantum theory and, therefore, derived from first principles of quantum theory. According to the above assumption the probability amplitude $\left\langle\psi_{2}\left|\tilde{S}\left(t_{2}, t_{1}\right)\right| \psi_{1}\right\rangle$ can itself be represented by the sum of amplitudes for each of the ways in which the subprocess with completely specified instants of the beginning and end of the interaction in a quantum system can be realized. However, some supplementary assumptions about the history of the system are needed. In the Feynman approach it is assumed that the history of the system can be represented by some path in space-time. In this case the amplitude $\left\langle\psi_{2}\left|\tilde{S}\left(t_{2}, t_{1}\right)\right| \psi_{1}\right\rangle$ can be in the form of integrals over all paths corresponding to processes in which the interaction begins at $t_{1}$ and ends at $t_{2}$. If, we also assume that each path gives a contribution is an exponential whose (imaginary) phase is the classical action (in units of $\hbar$ ) for this path (the second postulate of Feynman's theory) and substitute the expression obtained in this manner into Eq. (1), we arrive at Feynman's sum-over-paths formula for the transition amplitudes. At the same time, in Ref. [1] it has been shown that the use of the operator formalism of the canonical approach allows one to derive a relation for the amplitudes $\left\langle\psi_{2}\left|\tilde{S}\left(t_{2}, t_{1}\right)\right| \psi_{1}\right\rangle$, which can be used as a dynamical equation without resorting to the supplementary assumptions like the second postulate of Feynman's theory.

By using the operator formalism, we can represent the amplitudes $\left\langle\psi_{2}\left|U\left(t_{2}, t_{1}\right)\right| \psi_{1}\right\rangle$ by the matrix elements of the evolution operator $U\left(t, t_{0}\right)$, which must be uni- 
tary

$$
U^{+}\left(t, t_{0}\right) U\left(t, t_{0}\right)=U\left(t, t_{0}\right) U^{+}\left(t, t_{0}\right)=\mathbf{1}
$$

and satisfy the composition law

$$
U\left(t, t^{\prime}\right) U\left(t^{\prime}, t_{0}\right)=U\left(t, t_{0}\right), \quad U\left(t_{0}, t_{0}\right)=\mathbf{1}
$$

At the same time, $\tilde{S}\left(t_{2}, t_{1}\right)$ whose matrix elements are $\left\langle\psi_{2}\left|\tilde{S}\left(t_{2}, t_{1}\right)\right| \psi_{1}\right\rangle$ may be only an operator-valued generalized function of $t_{1}$ and $t_{2}$ [1], since only $U\left(t, t_{0}\right)=\mathbf{1}+\int_{t_{0}}^{t} d t_{2} \int_{t_{0}}^{t_{2}} d t_{1} \tilde{S}\left(t_{2}, t_{1}\right)$ must be an operator on the Hilbert space. Nevertheless, it is convenient to call $\tilde{S}\left(t_{2}, t_{1}\right)$ an "operator", using this word in generalized sense. In the case of an isolated system the operator $\tilde{S}\left(t_{2}, t_{1}\right)$ can be represented in the form [1]

$$
\tilde{S}\left(t_{2}, t_{1}\right)=\exp \left(i H_{0} t_{2}\right) \tilde{T}\left(t_{2}-t_{1}\right) \exp \left(-i H_{0} t_{1}\right)
$$

where $H_{0}$ is the free Hamiltonian.

As has been shown in Ref. [1], for the evolution operator given by (1) to be unitary and satisfy the composition law (3), the operator $\tilde{S}\left(t_{2}, t_{1}\right)$ must satisfy the following equation

$$
\left(t_{2}-t_{1}\right) \tilde{S}\left(t_{2}, t_{1}\right)=\int_{t_{1}}^{t_{2}} d t_{4} \int_{t_{1}}^{t_{4}} d t_{3}\left(t_{4}-t_{3}\right) \tilde{S}\left(t_{2}, t_{4}\right) \tilde{S}\left(t_{3}, t_{1}\right) .
$$

With this equation one can obtain the operators $\tilde{S}\left(t_{2}, t_{1}\right)$ for any $t_{1}$ and $t_{2}$, if the operators $\tilde{S}\left(t_{2}^{\prime}, t_{1}^{\prime}\right)$ corresponding to infinitesimal duration times $\tau=$ $t_{2}^{\prime}-t_{1}^{\prime}$ of interaction are known. It is natural to assume that most of the contribution to the evolution operator in the limit $t_{2} \rightarrow t_{1}$ comes from the processes associated with the fundamental interaction in the system under study. Denoting this contribution by $H_{i n t}\left(t_{2}, t_{1}\right)$, we can write

$$
\tilde{S}\left(t_{2}, t_{1}\right) \underset{t_{2} \rightarrow t_{1}}{\rightarrow} H_{i n t}\left(t_{2}, t_{1}\right)+o\left(\tau^{\varepsilon}\right)
$$

where $\tau=t_{2}-t_{1}$. The parameter $\varepsilon$ is determined by demanding that $H_{\text {int }}\left(t_{2}, t_{1}\right)$ must be so close to the solution of Eq. (5) in the limit $t_{2} \rightarrow t_{1}$ that this equation has a unique solution having the behavior (6) near the point $t_{2}=t_{1}$. Thus this operator must satisfy the condition

$$
H_{\text {int }}\left(t_{2}, t_{1}\right) \underset{t_{2} \rightarrow t_{1}}{\rightarrow} \int_{t_{1}}^{t_{2}} d t_{4} \int_{t_{1}}^{t_{4}} d t_{3} \frac{t_{4}-t_{3}}{t_{2}-t_{1}} H_{\text {int }}\left(t_{2}, t_{4}\right) H_{\text {int }}\left(t_{3}, t_{1}\right)+o\left(\tau^{\epsilon}\right) .
$$


Note that the value of the parameter $\epsilon$ depends on the form of the operator $H_{\text {int }}\left(t_{2}, t_{1}\right)$.

Within the GQD the operator $H_{\text {int }}\left(t_{2}, t_{1}\right)$ plays the same role as the interaction Hamiltonian in the ordinary formulation of quantum theory: It generates the dynamics of a system. Since this operator is a generalization of the interaction Hamiltonian, we call this operator the generalized interaction operator. If $H_{\text {int }}\left(t_{2}, t_{1}\right)$ is specified, Eq. (5) allows one to find the operator $\tilde{S}\left(t_{2}, t_{1}\right)$. Formula (1) can then be used to construct the evolution operator $U\left(t, t_{0}\right)$ and accordingly the state vector $|\psi(t)\rangle=\left|\psi\left(t_{0}\right)\right\rangle+\int_{t_{0}}^{t} d t_{2} \int_{t_{0}}^{t_{2}} d t_{1} \tilde{S}\left(t_{2}, t_{1}\right)\left|\psi\left(t_{0}\right)\right\rangle$ at any time $t$. Thus Eq. (5) can be regarded as an equation of motion for states of a quantum system. It should be noted that Eq. (5) is written only in terms of the operators $\tilde{S}\left(t_{2}, t_{1}\right)$, and does not explicitly contain operators describing the interaction in a quantum system. It is a relation for $\tilde{S}\left(t_{2}, t_{1}\right)$ which contains the contributions to the evolution operator from the processes with specified instants of the beginning and end of the interaction in the system. This relation is a unique consequence of the composition law (3) and the representation (1) expressing the Feynman superposition principle (the above assumption). For this reason the relation (5) must be satisfied in all the cases. A remarkable feature of this fundamental relation is that it works as a recurrence relation. For constructing the evolution operator, it is sufficient to know the contributions to this operator from the processes in which the duration time of interaction is infinitesimal, i.e., from the processes ruled by the fundamental interaction in the system. This makes it possible to use the fundamental relation (5) as a dynamical equation. Its form does not depend on the specific features of the interaction (the Schrödinger equation, for example, contains the interaction Hamiltonian). Since Eq. (5) must be satisfied in all the cases, it can be considered as the most general dynamical equation consistent with the current concepts of quantum theory. This generalized dynamical equation approaches the Schrödinger equation for appropriate boundary conditions.

By using (1), the evolution operator can be represented in the form [1]

$$
\begin{array}{r}
\left\langle n_{2}\left|U\left(t, t_{0}\right)\right| n_{1}\right\rangle=\left\langle n_{2} \mid n_{1}\right\rangle+\frac{i}{2 \pi} \int_{-\infty}^{\infty} d x \exp \left[-i\left(z-E_{n_{2}}\right) t\right] \\
\times \frac{\left\langle n_{2}|T(z)| n_{1}\right\rangle \exp \left[i\left(z-E_{n_{1}}\right) t_{0}\right]}{\left(z-E_{n_{2}}\right)\left(z-E_{n_{1}}\right)}
\end{array}
$$

where $z=x+i y$, and $y>0, n$ stands for the entire set of discrete and continuous variables that characterize the system in full, $|n\rangle$ are the eigenvectors of the free Hamiltonian $H_{0}$, and $\left\langle n_{2}|T(z)| n_{1}\right\rangle$ is defined by

$$
\left\langle n_{2}|T(z)| n_{1}\right\rangle=i \int_{0}^{\infty} d \tau \exp (i z \tau)\left\langle n_{2}|\tilde{T}(\tau)| n_{1}\right\rangle
$$


The equation of motion (5) is equivalent to the following equation for the $\mathrm{T}$ matrix [1]:

$$
\frac{d\left\langle n_{2}|T(z)| n_{1}\right\rangle}{d z}=-\sum_{n} \frac{\left\langle n_{2}|T(z)| n\right\rangle\left\langle n|T(z)| n_{1}\right\rangle}{\left(z-E_{n}\right)^{2}}
$$

with the boundary condition

$$
T(z) \underset{|z| \rightarrow \infty}{\rightarrow} i \int_{0}^{\infty} d \tau \exp (i z \tau) H_{\text {int }}^{(s)}(\tau)
$$

where $H_{\text {int }}^{(s)}\left(t_{2}-t_{1}\right)=\exp \left(-i H_{0} t_{2}\right) H_{\text {int }}\left(t_{2}, t_{1}\right) \exp \left(i H_{0} t_{1}\right)$ is the generalized interaction operator in the Schrödinger picture. As has been shown in Ref. [1], the dynamics governed by Eq. (5) is equivalent to the Hamiltonian dynamics in the case where the generalized interaction operator is of the form

$$
H_{\text {int }}\left(t_{2}, t_{1}\right)=-2 i \delta\left(t_{2}-t_{1}\right) H_{I}\left(t_{1}\right)
$$

Here $H_{I}\left(t_{1}\right)$ is the interaction Hamiltonian in the interaction picture. In this case the evolution operator given by (1) satisfies the Schrödinger equation. The delta-function in (11) emphasizes the fact that in this case the fundamental interaction is instantaneous. At the same time, Eq. (5) permits the generalization to the case where the interaction generating the dynamics of a system is nonlocal in time [1]. In Ref. [1,9] this point was demonstrated on exactly solvable models.

Let us consider an atom with its radiation field as an open quantum system interacting with its surroundings. We will consider the surroundings as a whole identity, referred as the thermal bath. Let the bath be non-GaussianMarkovian, i.e., the time scale $t_{c}$ of the bath with respect to the system dynamics is very short but $t_{c} \neq 0$. Further, we assume that the evolution operator $U_{o p}\left(t_{2}, t_{1}\right)$ defined on the subspace $H_{o p}$ describing the open system satisfies the semi-group law

$$
U_{o p}\left(t_{2}, t\right) U_{o p}\left(t, t_{1}\right)=U_{o p}\left(t_{2}, t_{1}\right), \quad t_{2} \geq t \geq t_{1}
$$

and $\left\|U_{o p}\left(t_{2}, t_{1}\right)\right\| \leq 1$ for all $t_{2}>t_{1}$. Obviously, due to a loss of probability from the open system, the evolution operator is not unitary. Thus open quantum systems are unstable. An approach to the theory of unstable quantum systems based on the semi-group law has been developed in the works of Williams [19], Sinha [20] and Horwitz et all [21] (for reviews see Ref. [22]). It is assumed that the evolution operator $U_{o p}\left(t_{2}, t_{1}\right)$ satisfies the semi-group law and is strongly continuous. If this assumption can be seen as fulfilled from the physical point of 
view, the above approach provides a fundamental description of the dynamics of open quantum systems. In fact from Stone's theorem [23] it follows that in the case of a closed system the assumption that the evolution operator satisfies the composition law (3), and is unitary and strongly continuous is equivalent to the assumption that the evolution of a quantum system is governed by the Schrödinger equation [24]. This result can be generalized to the case of open quantum systems. However, the semi-group law (12) is not satisfied in general. It is satisfied only in the case when at any time during the evolution the system remains in a pure state belonging to the subspace $H_{o p}$. But this, of course, does not take place in the general case. This fact by itself allows us to conclude that a semi-group law cannot hold for the evolution operator on the Hilbert space $H_{o p}$, while the evolution operator acting on the Banach space of density operators satisfies the semi- group law in the general case. Thus the theory has to be reformulated in the language of the density operator. This program has been realized in the Lindblad theory. This theory is founded on the same assumptions as the above approach translated into the language of the density operator, i.e., on the assumptions that the evolution operator on the Banach space of density operators satisfies the semi-group law and is continuous. However in this theory the interaction generating the dynamics of a quantum system is instantaneous. However, the interaction with the nonGaussian-Markovian bath must be nonlocal in time.

The GQD allows the extension of quantum theory to the case when the evolution operator is not continuous, and precisely in this case the interaction in a system is nonlocal in time [1]. As for the validity of the semi-group law (11), the GQD deals with the probability amplitudes in the spirit of the Feynman approach, and the representation (5) can be written for probability amplitudes of any event concerning the evolution of the system. In this case the operator $U\left(t_{2}, t_{1}\right)$ should be defined as an operator whose matrix elements represent these amplitudes. In the case under consideration we can define the matrix element $\left\langle\psi_{2}\left|U_{o p}\left(t_{2}, t_{1}\right)\right| \psi_{1}\right\rangle$ as the probability amplitude that if at time $t_{1}$ the system was in the state $\left|\psi_{1}\right\rangle \in H_{o p}$, then all the time between $t_{1}$ and $t_{2}$ the system will be in states belonging to the subspace $H_{o p}$, and at time $t_{2}$ the system will be found in the state $\left|\psi_{2}\right\rangle \in H_{o p}$. The fact that in the time interval $\left(t_{2}, t_{1}\right)$ the system is only in states belonging $H_{o p}$ means that all the time between $t_{1}$ and $t_{2}$ we deal with the atom but not with its decay products (nuclear or ions and electrons). Thus the matrix elements $\left\langle\psi_{2}\left|U_{o p}\left(t_{2}, t_{1}\right)\right| \psi_{1}\right\rangle$ represent some alternative way of realization of the evolution process. The operator $U_{o p}\left(t_{2}, t_{1}\right)$ defined in this way is not equivalent to the ordinary evolution operator defined on the space $H_{o p}$. Since the operator $U_{o p}\left(t_{2}, t_{1}\right)$ satisfies the semi-group law (12), we can describe the system in terms of state vectors on 
the space $H_{o p}$. The operator $U_{o p}\left(t, t_{0}\right)$ can then be represented in the form

$$
\left\langle\psi_{2}\left|U_{o p}\left(t, t_{0}\right)\right| \psi_{1}\right\rangle=\left\langle\psi_{2} \mid \psi_{1}\right\rangle+\int_{t_{0}}^{t} d t_{2} \int_{t_{0}}^{t_{2}} d t_{1}\left\langle\psi_{2}\left|\tilde{S}_{o p}\left(t_{2}, t_{1}\right)\right| \psi_{1}\right\rangle
$$

where the operator $\tilde{S}_{o p}\left(t_{2}, t_{1}\right)$ describes the contribution to the operator $U_{o p}\left(t, t_{0}\right)$ from the process in which the interaction in the system begins at time $t_{1}$ and ends at time $t_{2}$. If the condition of the bath does not change in time, the evolution of the open system under study must be invariant in time, and consequently the operator $\tilde{S}_{o p}\left(t_{2}, t_{1}\right)$ can be represented in the form $\tilde{S}_{o p}\left(t_{2}, t_{1}\right)=$ $\exp \left(i H_{0} t_{2}\right) \tilde{T}\left(t_{2}-t_{1}\right) \exp \left(-i H_{0} t_{1}\right)$, and the corresponding $T(z)$ is defined by (9). Here we use the same notation for the operators $\tilde{T}(\tau)$ as in the case of closed systems.

The operator $U_{o p}\left(t_{2}, t_{1}\right)$ characterizes the evolution process in the open quantum system. As example, let us consider a two-level atom. The matrix element $\left\langle\mathbf{k}, \varepsilon_{\lambda}, 1\left|U_{o p}\left(t_{2}, t_{1}\right)\right| 2\right\rangle$, is the probability amplitude that if at time $t_{1}$ the system was in the state $|2\rangle$, then at time $t_{2}$ the system will be found in the state $\left|\mathbf{k}, \varepsilon_{\lambda}, 1\right\rangle$. Here $|2\rangle$ and $|1\rangle$ denote the excited and ground states of the atom, respectively, $\mathbf{k}$ is the momentum of a photon and $\varepsilon_{\lambda}$ being the photon polarization. If the ground state $|1\rangle$ of the atom can be regarded as stable, then $\sum_{\lambda=1,2}\left|\left\langle\mathbf{k}, \varepsilon_{\lambda}, 1\left|U_{o p}(\infty, 0)\right| 2\right\rangle\right|^{2} d \omega$ is the probability of a photon being emitted by a single atom with energy in the interval $(\omega, \omega+d \omega)$. From this it follows that the spectral-line profile is described by the formula

$$
\frac{d W_{21}(\omega)}{d \omega}=\sum_{\lambda=1,2}\left|\left\langle\mathbf{k}, \varepsilon_{\lambda}, 1\left|U_{o p}(\infty, 0)\right| 2\right\rangle\right|^{2}
$$

Thus the operator $U_{o p}\left(t_{2}, t_{1}\right)$ determines the broadening of spectral lines of atoms caused by the interaction with their environment. Let the generalized interaction operator describing the interaction of the atom with its own radiation field and the bath be of the form

$$
H_{i n t}^{(s)}(\tau)=-2 i \delta(\tau) H_{I}^{(v)}+H_{i n t}^{(b)}(\tau)
$$

with

$$
H_{I}^{(v)}=\int d^{3} x j_{\mu}(\mathbf{x}, 0) A^{\mu}(\mathbf{x}, 0)
$$

Here $j_{\mu}(x)$ is the current density operator, $A_{\mu}(x)$ is the electromagnetic field 
potential and $H_{i n t}^{(b)}(t)$ is as follows:

$$
\left\{\begin{array}{r}
\left\langle l\left|H_{i n t}^{(b)}(\tau)\right| j\right\rangle=(f(\tau)-2 i \Lambda \delta(\tau)) \delta_{l 2} \delta_{j 2} \\
\left\langle\mathbf{k}, \varepsilon_{\lambda}, l\left|H_{i n t}^{(b)}(\tau)\right| j\right\rangle=\psi(\mathbf{k}) f(\tau) \delta_{l 1} \delta_{j 2} \\
\left\langle l\left|H_{i n t}^{(b)}(\tau)\right| \mathbf{k}, \varepsilon_{\lambda}, j\right\rangle=\psi^{*}(\mathbf{k}) f(\tau) \delta_{l 2} \delta_{j 1} \\
\left\langle\mathbf{k}_{2}, \varepsilon_{\lambda_{2}}, l\left|H_{i n t}^{(b)}(\tau)\right| \mathbf{k}_{1}, \varepsilon_{\lambda_{1}}, j\right\rangle=\psi\left(\mathbf{k}_{2}\right) \psi^{*}\left(\mathbf{k}_{1}\right) f_{1}(\tau) \delta_{l 1} \delta_{j 1}
\end{array}\right.
$$

where the form factor $\psi(\mathbf{k})$ is chosen as $\psi(\mathbf{k})=c_{1}\left(d^{2}+k^{2}\right)^{-\frac{1}{2}}$, and $\Lambda, c_{1}$ and $d$ being some constants. The function $f(\tau)$, characterizing the nonlocality in time of the interaction, is not arbitrary since the interaction operator (15) must satisfy the condition (7). For this condition to be satisfied, the function $f(\tau)$ must be of the form

$$
f(\tau)=-\frac{i}{2 \pi} \int_{-\infty}^{\infty} \exp (-i z \tau)\left(\frac{b_{1}}{\ln (-z)}+\frac{b_{2}}{\ln ^{2}(-z)}\right) d z
$$

where $b_{1}=-\frac{1}{4 \pi c_{1}^{2}}$ and only one parameter $b_{2}$ is free. The operator $H_{I}^{(v)}$ describes the interaction of an isolated atom with the electromagnetic field, while the operator $H_{\text {int }}^{(b)}(\tau)$ describes the interaction in which the bath manifests itself.

The main assumption that we have used in choosing the form of the operator $H_{i n t}^{(b)}(\tau)$ is that its matrix element $\left\langle 2\left|H_{i n t}^{(b)}(\tau)\right| \mathbf{k}, \varepsilon_{\lambda}, 1\right\rangle$ should be of the form $\left\langle 2\left|H_{\text {int }}^{(b)}(\tau)\right| \mathbf{k}, \varepsilon_{\lambda}, 1\right\rangle=\psi^{*}(\mathbf{k}) f(\tau)$. As has been shown in [1], there is a oneto-one correspondence between the form of the interaction operator and the ultraviolet (UV) behavior of the matrix elements of the evolution operator as functions of momenta of photons: If this behavior satisfies the requirements of conventional quantum theory, then $H_{\text {int }}\left(t_{2}, t_{1}\right)$ must necessarily be of the form (11), i.e., the interaction must be instantaneous, and, on the contrary, if this behavior is "bad" and leads to UV divergences, then $H_{\text {int }}\left(t_{2}, t_{1}\right)$ must be nonlocal in time. In the case of separable interactions the above behavior and hence the form $H_{\text {int }}\left(t_{2}, t_{1}\right)$ are determined by the UV behavior of the corresponding form factors. Let the form factor has the following UV behavior $\psi(\mathbf{k}) \sim \frac{1}{|\mathbf{k}|^{\alpha}}$. In the case $\alpha>1$, the function $f(\tau)$ must be of the form $f(\tau)=A \delta(\tau)$, where $A$ being some constant, i.e., the interaction is instantaneous. In the case $0<\alpha \leq 1$ (the restriction $\alpha>0$ is necessary for the generalized dynamical equation (5) to have the mathematical meaning) the function $f(\tau)$ has no such singularity as the delta-function at $\tau=0$, and hence the interaction is nonlocal in time. Since the interaction of the atom with its surroundings is nonlocal in time, we have to use a form factor corresponding to the case $0<\alpha \leq 1$. The interaction is nonlocal in time for any $0<\alpha \leq 1$. 
However the case $\alpha=1$ seems to be most natural. In fact, in this case the UV behavior of the form factors gives rise to the logarithmic divergences in the Born series, i.e., to the one of the fundamental divergences of QED. The simplest form factor with such a UV behavior is of the form $\psi(\mathbf{k})=c_{1}\left(d^{2}+k^{2}\right)^{-\frac{1}{2}}$ (the parameter $d$ is needed in order to resolve the problem of the infrared divergences). For these reasons we have chosen the form factor in this form. From the condition (7) it follows that the operator $H_{i n t}^{(s)}(\tau)$ must also describe the processes of the atom-photon scattering, and the corresponding matrix element must be of the form $\left\langle\mathbf{k}_{2}, \varepsilon_{\lambda_{\mathbf{2}}}, 1\left|H_{\text {int }}^{(s)}(\tau)\right| \mathbf{k}_{1}, \varepsilon_{\lambda_{1}}, 1\right\rangle=\psi\left(\mathbf{k}_{2}\right) \psi^{*}\left(\mathbf{k}_{1}\right) f(\tau)$ with the same duration-time function $f(\tau)$ given by $(17)$. Note that there is only one free parameter $b_{2}$ in this function. Thus the form of the interaction operator is determined by the UV behavior of the form factor $\psi(\mathbf{k})$. However, this concerns only the nonlocal part of the interaction operator, and one may supplement the interaction operator by an instantaneous part [9]. The interaction operator $H_{\text {int }}^{(b)}(\tau)$ also contains the instantaneous term. But in our model it describes only the interaction of the atom in the excited state. We do not take into account this interaction of the atom in the ground state, because we assume that the possibility for a photon decay is very small when the atom is in the ground state, and hence the interaction can lead only to an energy shift of this state.

Let us consider the case where $c_{1}=0$, i.e., where the interaction of the atom with its environment is reduced to the instantaneous interaction being described by the potential $\langle i|V| j\rangle=\Lambda \delta_{i 2} \delta_{j 2}$. We will assume that $\langle i|V| j\rangle$ is such that the interaction of the atom with the radiation field can be considered as a small perturbation, and hence the problem can be solved by expanding in powers of $H_{I}^{(v)}$. In the first order, for $\left\langle\mathbf{k}, \varepsilon_{\lambda}, 1|T(z)| 2\right\rangle$, we have

$$
\left\langle\mathbf{k}, \varepsilon_{\lambda}, 1\left|T^{(1)}(z)\right| 2\right\rangle=\frac{\left\langle\mathbf{k}, \varepsilon_{\lambda}, 1\left|H_{I}^{(v)}\right| 2\right\rangle\left(z-E_{2}\right)}{z-E_{2}-\Lambda} .
$$

Substituting this expression into (8) and using (14), we then get

$$
\frac{d W_{21}(\omega)}{d \omega}=A \omega \sum_{\lambda=1,2} \int d \Omega_{k}\left|\frac{\left\langle\mathbf{k}, \varepsilon_{\lambda}, 1\left|H_{I}^{(v)}\right| 2\right\rangle}{E_{1}+\omega-E_{2}-\Delta E_{2}+\frac{i}{2} \Gamma_{2}}\right|,
$$

where $\Delta E_{2}=\operatorname{Re} \Lambda, \Gamma_{2}=-2 \operatorname{Im} \Lambda$, and $A$ is a renormalization factor. In the model under consideration $\langle i|V| j\rangle$ can be considered as the corresponding matrix elements of an potential which parametrize effects of impacts of the atom with particles from its surroundings, and hence $\Delta E_{2}$ and $\Gamma_{j}$ can be interpreted, accordingly, as the impact line shift and width.

Thus in the case where the interaction with its surroundings is instantaneous, our model yields the same results as the theory of impact broadening of spec- 
tral lines. At the same time, our model allows the extension to the case where the interaction of the atom with its surroundings results not only in radiationless transitions between the excited and ground states, but also in the emission of photons. Such processes are generally nonlocal in time, and the parameter $c_{1}$ cannot be zero. Let us now solve the problem in this case. We will assume that the interaction of the atom with its surroundings is such that we can restrict ourselves to the zero order in powers of $H_{I}^{(v)}$. In this case the dynamical equation (10) can be exactly solved, and the corresponding solution is

$$
\left\{\begin{array}{r}
\langle l|T(z)| j\rangle=t_{22}(z) \delta_{l 2} \delta_{j 1} \\
\left\langle\mathbf{k}, \varepsilon_{\lambda}, 2|T(z)| 1\right\rangle=\psi(\mathbf{k}) t_{12}(z) \\
\left\langle 2|T(z)| \mathbf{k}, \varepsilon_{\lambda}, 1\right\rangle=\psi^{*}(\mathbf{k}) t_{21}(z) \\
\left\langle\mathbf{k}_{2}, \varepsilon_{\lambda_{2}}, 1|T(z)| \mathbf{k}_{1}, \varepsilon_{\lambda_{1}}, 1\right\rangle=\psi\left(\mathbf{k}_{2}\right) \psi^{*}\left(\mathbf{k}_{1}\right) t_{11}(z)
\end{array}\right.
$$

where

$$
\begin{aligned}
& t_{22}(z)=\frac{\left(\Lambda+T_{1}(z)\right)\left(z-E_{2}\right)}{z-E_{2}-\Lambda-T_{1}(z)}, \quad t_{11}(z)=\frac{T_{1}(z)\left(z-E_{2}-\Lambda\right)}{z-E_{2}-\Lambda-T_{1}(z)}, \\
& t_{21}(z)=t_{12}(z)=\frac{T_{1}(z)\left(z-E_{2}\right)}{z-E_{2}-\Lambda-T_{1}(z)},
\end{aligned}
$$

with

$$
T_{1}(z)=\frac{-\frac{1}{4 \pi c_{1}^{2}} \frac{d^{2}+\left(z-E_{1}\right)^{2}}{\left(z-E_{1}\right)^{2}}}{-\pi i+\ln \left(\frac{z-E_{1}}{d_{0}}\right)+\frac{d^{2} \ln \frac{d}{d_{0}}}{\left(z-E_{1}\right)^{2}}-\frac{\pi d}{2\left(z-E_{1}\right)}-\frac{b_{2}\left(d^{2}+\left(z-E_{1}\right)^{2}\right)}{b_{1}\left(z-E_{1}\right)^{2}}},
$$

where $d_{0}=1 \mathrm{eV}$. By using this formula and Eq. (8) we can construct the operator $U_{o p}\left(t, t_{0}\right)$. For example, we can obtain the matrix element $\left\langle\mathbf{k}, \varepsilon_{\lambda}, 1|U(\infty, 0)| 2\right\rangle$, which, as it follows from (14), determines the broadening of the spectral line of the atom. By using (14) and the above expression for the T matrix, we then get

$$
\frac{d W_{21}(\omega)}{d \omega}=\frac{1}{2 \pi} \frac{A f(\omega)}{\left(\omega+E_{1}-E_{2}-\Delta E(\omega)\right)^{2}+\frac{1}{4} \Gamma^{2}(\omega)},
$$

where

$$
\begin{aligned}
& f(\omega)=\frac{d^{2}+\omega^{2}}{4 \pi c_{1}^{2} \omega^{4}}\left(\pi^{2}+\left[\ln \frac{\omega}{d_{0}}+\frac{d^{2}}{\omega^{2}} \ln \frac{d}{d_{0}}-\frac{\pi d}{2 \omega}-\frac{b_{2}\left(d^{2}+\omega^{2}\right)}{b_{1} \omega^{2}}\right]^{2}\right)^{-1} \\
& \Delta E(\omega)=\operatorname{Re} \Lambda-f(\omega)\left(\omega^{2} \ln \frac{\omega}{d_{0}}+d^{2} \ln \frac{d}{d_{0}}-\frac{\pi}{2} d \omega-\frac{b_{2}}{b_{1}}\left[d^{2}+\omega^{2}\right]\right)
\end{aligned}
$$



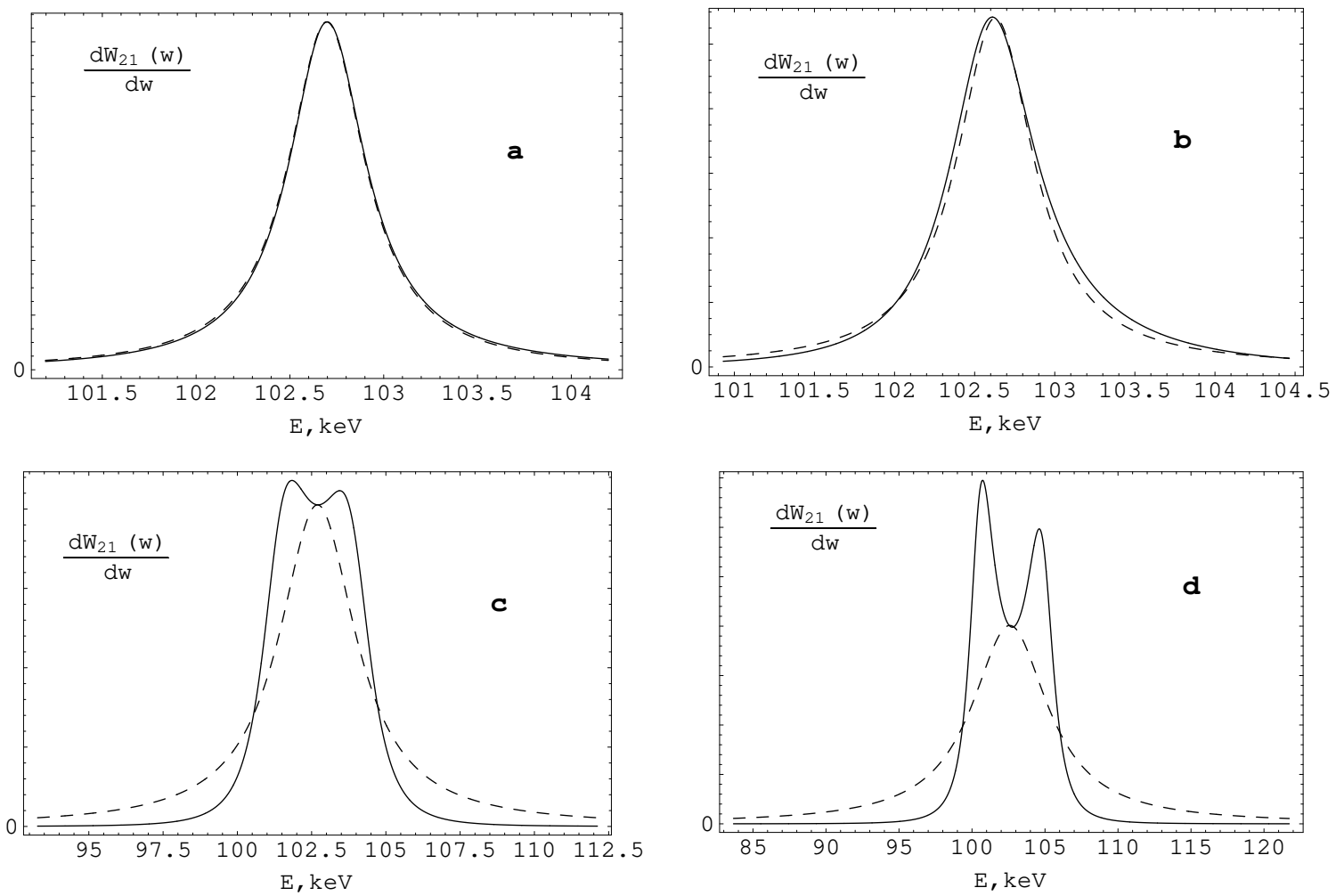

Fig. 1. The profile of the spectral line $2 P_{\frac{1}{2}} \rightarrow 1 S_{\frac{1}{2}}$ of the hydrogen-like atom, $Z=92$, $E_{2}-E_{1}=102697 \mathrm{eV}$. The dashed line represent the corresponding Lorentzian line for the parameters given in Table 1.

$$
\Gamma(\omega)=2\left(\operatorname{Im} \Lambda+\pi \omega^{2} f(\omega)\right) .
$$

This formula describes the spectral line profile of the atom in our model. In order to find out how the nonlocality in time of interaction of the atom with its surroundings can influence on the spectral line broadening, we have made the calculations of the profile (23) for various values of the parameters of the model. The results of the calculations are given in Fig.1.

For certain values of the parameters the nonlocality of interaction has very significant effects on the spectral line broadening. Fig.1(c,d), for example, shows that in some cases such a nonlocality of the interaction gives rise to the splitting of the spectral line of the atom.

To summarize and to conclude, we hade shown that the GQD can be used to describe, in a natural way, the evolution of an open quantum system whose interaction with its environment is nonlocal in time. We have defined the operator $U_{o p}\left(t_{2}, t_{1}\right)$, in terms of which the dynamics of open quantum systems can be described in the same way as the dynamics of closed systems. In the case when the open system is an atom with its radiation field this operator determines the broadening of spectral-line profiles of atoms caused by a 
Table 1

The parameters of the interaction operator (15)

\begin{tabular}{|l|c|c|c|c|}
\hline Figs., & $\Lambda, \mathrm{eV}$ & $c_{1}^{2}, \mathrm{eV}^{-1}$ & $d, \mathrm{eV}$ & $b_{2}, \mathrm{eV}$ \\
\hline a & $250 \imath$ & 25 & $1.25 \cdot 10^{7}$ & -0.052 \\
$\mathrm{~b}$ & $250 \imath$ & 9 & $1.25 \cdot 10^{7}$ & -0.144 \\
$\mathrm{c}$ & $300 \imath$ & 0.25 & $1.15 \cdot 10^{7}$ & -5.170 \\
d & $250 \imath$ & 0.09 & $1.05 \cdot 10^{7}$ & -14.281 \\
\hline
\end{tabular}

nonlocal-in-time interaction with its environment. By using an exactly solvable model, we found that the nonlocality in time of the interaction of the atom with its surroundings can have substantial effects on the spectral line broadening. As it follows from our calculations, for the most values of the parameters the broadening of the spectral line profile does not differ from the Lorentzian line, i.e., the effects of nonlocality in time of interaction on the broadening of the spectral line are negligible. However, there is a narrow interval of values of the parameters for which the broadening of the spectral line differs profoundly from the Lorentzian, and the spectral lines can even split. This means that some resonance condition of the bath can exists which has the effect that the spectral lines split. Our results can be considered as a prediction of the above effect.

\section{References}

[1] R.Kh. Gainutdinov, J. Phys. A 32 (1999) 5657.

[2] R.P. Feynman, Rev. Mod. Phys. 20 (1948) 367.

[3] R. Feynman and A. Hibbs, Quantum Mechanics and Path Integrals. McGraw-Hill, New York, 1965.

[4] S. Weinberg, Physica A. 96327 (1979).

[5] E. Witten, Nucl. Phys. B. 122109 (1977).

[6] S. Weinberg, Phys. Lett. B. 251288 (1990); Nucl. Phys. B. 3633 (1991).

[7] S.R. Beane, P.F. Bedaque, W.C. Haxton, D.R. Phillips and M.L. Savage, nucl-th/0008064.

[8] R.Kh. Gainutdinov and A.A. Mutygullina, Phys. Rev. C. 66, 014006 (2002).

[9] R.Kh. Gainutdinov and A.A. Mutygullina, Yad. Fiz.. 62 (1999) 2061.

[10] R.Kh. Gainutdinov and A.A. Mutygullina, Yad. Fiz.. 65, 1456 (2002).

[11] R.Kh. Gainutdinov and A.A. Mutygullina, Mesons and Light Nuclei, edited by J. Adam, P. Byžovský, J. Mareš (American Institute of Physics, New York, 2001) 603, 291 (2001).

[12] G. Lindblad, Rep. Math. Phys. 10 (1976) 393.

[13] G. Lindblad, Commun. Math. Phys. 48 (1976) 119.

[14] A. Sandulescu and H. Scutaru, Ann. Phys. (N.Y.) 173 (1987) 277. 
[15] E. Stefanescu, A. Sandulescu, W. Greiner, and W. Scheid, Phys. Rev. C 53 (1996) 3014.

[16] G.G. Adamian, N.V. Antonenko, W. Scheid, Phys. Lett. A 244 (1998) 482.

[17] G.G. Adamian, N.V. Antonenko, W. Scheid, Phys. Lett. A 260 (1999) 39.

[18] A. Sudbery, Quantum Mechanics and the Particles of Nature. Cambridge University Press, Cambridge, 1986.

[19] D.N. Williams, Commun. Math. Phys. 21 (1971) 314.

[20] K. Sinha, Helv. Phys. Acta 45 (1972) 619.

[21] L.P. Horwitz, J.A. La Vita and J.P. Marchand, J. Math. Phys. 12 (1971) 2537.

[22] L. Fonda, G.C, Ghirardi and A. Rimini, Rep. Prog. Phys. 41 (1978) 587.

[23] M. Reed and B. Simon, Methods of modern mathematical physics. 1. Functional analysis . Academic Press, New York London, 1972.

[24] J.-P. Antoine, J. Math. Phys. 10 (1969) 53. 